\title{
Emergence!
}

\section{(The Ontological Conjecture)}

\section{Dedicated to Henry Siggins Leonard}

\section{Ronald Preston Phipps*}

Ours is a vacuumless universe Character and Potential pervade The spatio-temporal manifold!

The manifold is discretely divided As the ancients foretold By atomicity, by discreteness amid continuity!

Atomicity embedded within atomic occasions Events emerging as a confluence of Antecedent events!

The character and relations within that nexus Constituent of an occasion's causal past Determine the characteristics and relations Which ingress within the atomic event!

As character ingresses into an atomic occasion, Bounded by spatial magnitude and temporal duration, The character and potentiality Within the event's causal future Emerge, Begat as an occasion's progeny.

Co-Director, International Center for Process Philosophy, Science and Education 
Generating diverse causal futures,

Overlapping, interlaced, interacting!

The Community of Being

Omnipresent, The Community of Being

Continuous and infinite, borderless and boundless!

Puffs of becoming perpetually emerging perpetually perishing Within the Flux!

The void exists merely in Abstraction -

The dynamic interplay

Of the Perpetual Flux characterizes the concrete!

No mythical Singularity exists

Devoid of character, structure, origin, history and environment!

The expansion, contraction, contortion, rhythm of Cosmic Epochs Emerging, changing and perishing

Within the Cosmic Community represent the cosmology of our Sage!

To understand the Real

The Sacred Trinity,

Philosophy, Physics and Poetry

Must by the Academy of Artists

Be woven into a new conceptual Tapestry!

Such is the causal orderliness that governs the transition

From the past to the present,

An orderliness in the absence of which

Teleology, Morality and Aesthetics would never appear!

Democratus and Lucretius planted the seeds of atomicity Within the minds of philosophers and physicists, A mode of atomicity whose roots have penetrated Deeply and broadly the consciousness of all science! Atomicity and Reductionism were the yin/yang of physics 
Until a new sage appeared!

Atomicity, this sage taught us,

Does not imply that qualitative variety is denied, squeezed out From the micro world!

Such is a false, hollow assumption restricting our imagination!

The world of the quantum

Does not confine atomic occasions to infinite geometric variety

Merely in the modes of aggregation of sameness of constituents!

Open our minds

To see the deep dynamism of existence!

A transformation of Ontological Perspective is imperative.

Accelerators as cauldrons of dissociation

To Fields as cauldrons of Creative Synthesis

And emergence of Atomic Occasions within a Sea of Fields

Within the Infinite and Eternal Manifold of Becoming!

Descartes' Illusion of Separate Realms, reality bifurcated, Replaced by Fields generating the ingression of diverse attributes Physical and Mental within Atomic Occasions

Emerging from Creative Synthesis

And generated by Fields

And complex Modes of Aggregations!

Cauldrons of Ontological Dissociation

Transformed into

Cauldrons of Creative Synthesis omnipresent in

Fields of Becoming!

Processes of Dissociation and

Processes of Creative Synthesis are jointly necessary

To understand Emergence 
As the Universal Phenomena within the Fabric of Being!

Emergence of Atomic Occasions is guided by Field Equations Describing the patterns by which the past transitions

To the present and the present is lured to its future!

If Field Equations generating new modes of atomicity Are amenable to infinite solutions

Then the vacuumless universe

Is infinite qualitatively and quantitatively!

A new form of atomicity

Is postulated in the Ontological Conjecture

From heaven Master Alfred and Disciple Leonard

With Socrates and Plato

Look down upon our tiny Planet and smile!

Openness overwhelms closedness.

Orderliness and infinite qualitative variety

Are harmonized in a new Synthesis, a deeper Integration!

Beauty and wonder

Prevail.

The ancient atomicity of sameness is overcome

By the atomicity of variety

Husbanded by Field Equations

And the Orderliness of Nature itself!

As dogmatism and orthodoxy are superseded,

A new journey of inquiry

Guided by the perspective of process and organism

Will commence and last 10,000 years and more!

Reductionism has played its historic Role

But Relentless Reductionism

From the kingdom of ontology and cosmology 
Is now banished!

And the Sages

More broadly smile!

To be absent for more than two millennia -

What is that to truth and beauty?

Orderliness and Variety

Within the Small, the Large and the Complex

In conceptual harmony will Reign;

Light over the Dark will finally prevail!

*Prof. Henry Siggins Leonard was the past President of the American Philosophical Association. At Harvard Leonard was Whitehead's student and colleague for a decade and his Personal Assistant for 6 years during the period Whitehead wrote and taught Process and Reality and other major philosophic works. 